\title{
The impact of delayed time to first CT head in traumatic brain injury
}

\author{
Morgan Schellenberg $^{1} \cdot$ Elizabeth Benjamin ${ }^{1} \cdot$ Natthida Owattanapanich $^{1} \cdot$ Kenji Inaba $^{1} \cdot$ Demetrios Demetriades $^{1}$
}

Received: 17 April 2020 / Accepted: 15 June 2020 / Published online: 25 June 2020

(c) Springer-Verlag GmbH Germany, part of Springer Nature 2020

\begin{abstract}
Purpose Trauma team activation (TTA) criteria trigger early mobilization of resources for the sickest trauma patients. Patients with moderately depressed GCS who do not trigger the highest level activation are at risk for adverse outcomes, potentially from delayed time to intervention. The study objective was to define the impact of time to first CT Head (CTH) on outcomes among blunt trauma patients with moderately depressed GCS.

Methods Patients from the Trauma Quality Improvement Program (TQIP) databank (2013-2016) with first ED GCS 9-12 were included. Transfers, penetrating mechanisms, death $<24 \mathrm{~h}$, AIS $=6$ in any body region, and patients with severe associated injuries were excluded. Study groups were defined by time to first CTH after ED arrival: immediate $(\leq 1 \mathrm{~h})$ vs. delayed (1-6 h). Primary outcomes were time to neurosurgical intervention and time to ED discharge.

Results After exclusions, 4997 patients were identified. Of these, $79 \%(n=3,954)$ underwent immediate CTH and $21 \%$ $(n=1,043)$ had delayed CTH. Median GCS was 11 [10-12] in both groups and there was no difference in median Head AIS (4 [3-4] vs. 4 [3-4], $p=0.586)$. Time to craniotomy and ICP monitor insertion were longer in the delayed group $(4.2 \mathrm{~h}$

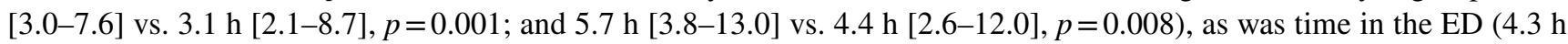
[2.7-6.5] vs. $2.1 \mathrm{~h}[1.2-3.7], p<0.001)$. There was no difference in need for craniotomy (11\% vs. $10 \%, p=0.287)$, need for ICP monitor ( $12 \%$ vs. $12 \%, p=0.899)$, or mortality $(11 \%$ vs. $9 \%, p=0.160)$. On multivariate analysis, age $>65$ (OR 2.813 , $p<0.001$ ), $\mathrm{SBP}<90 \mathrm{mmHg}$ (OR 2.934, $p<0.001$ ), ED intubation (OR 1.486, $p=0.001$ ), and Head AIS scores of 4 (OR $1.884, p<0.001)$ and 5 (OR $6.729, p<0.001)$ were independently associated with death.

Conclusions Immediate CTH for blunt trauma patients with moderately depressed GCS decreases time to intervention and reduces ED time. A protocol to shorten time to CTH may be beneficial for both patients and hospitals.
\end{abstract}

Keywords Traumatic brain injury · Computed tomography of the head · Undertriage · Time to intervention · Trauma

\section{Background}

The American College of Surgeons (ACS) Committee on Trauma (COT) delineates a set of trauma team activation (TTA) criteria, which are intended to identify the sickest trauma patients in the prehospital setting to allow for early mobilization of personnel and resources prior to patient arrival in the emergency department (ED) [1]. These criteria are based on physiologic variables and mechanism of injury characteristics. Currently, patients with traumatic

Morgan Schellenberg

morgan.schellenberg@med.usc.edu

1 Division of Trauma and Surgical Critical Care. LAC + USC Medical Center, University of Southern California, 2051 Marengo Street, Inpatient Tower, C5L100, Los Angeles, CA 90033, USA brain injuries (TBI) trigger TTA when the field Glasgow Coma Scale (GCS) score is $<9$. However, a recent study of severely injured patients who did not meet standard TTA criteria demonstrated that a GCS score $<11$ identified a population at risk for mortality and need for emergent intervention [2].

It is important to define at-risk patients who are not captured by the existing TTA criteria in order to identify additional patient populations who may also benefit from early mobilization of personnel and resources. One of the potential benefits of TTA is a streamlined diagnostic process, whereby patients who meet TTA criteria are given expedited access to computed tomography (CT) scanning. In turn, this may facilitate timely treatment. Therefore, patients who do not meet TTA criteria may be at risk for adverse outcomes because of prolonged time to investigations and intervention. 
The objective of this study was to determine the impact of time to first CT Head (CTH) on outcomes among patients with moderately depressed GCS on arrival to the ED. The hypothesis was patients who do not undergo immediate $\mathrm{CTH}$ are at risk for poor outcomes as a result of delayed time to investigations and interventions.

\section{Methods}

In this retrospective observational study, all patients with an initial GCS score of 9-12 in the ED were included (January 1, 2013-December 31, 2016). Patients were identified from the Trauma Quality Improvement Program (TQIP), a subset of the National Trauma Data Bank (NTDB). To be captured by TQIP, patients must present to one of $>700$ participating US Level I or II trauma centers with age $>16$ years and an Abbreviated Injury Scale (AIS) score $>2$ in at least one body region. Institutional Review Board approval was sought from the University of Southern California and exemption was granted with a waiver of informed consent.

Exclusion criteria were severe associated injuries, defined by AIS $\geq 3$ in the spine, chest, abdomen, or extremities; transfer from outside hospital; arrival without signs of life; death in the ED or $<24 \mathrm{~h}$ of admission, in order to exclude those with unsalvageable injuries; penetrating injuries or unspecified mechanism of injury; AIS $=6$ in any body region; time to first $\mathrm{CTH}>6 \mathrm{~h}$ after $\mathrm{ED}$ arrival; and missing procedure codes, procedure times, or discharge disposition.

Data collection variables included patient demographic data (age, gender, and comorbidities), clinical data [prehospital and first ED systolic blood pressure (SBP, mmHg), heart rate (HR, beats per minute (bpm)), and GCS]; injury data [mechanism of injury, level of trauma center, Injury Severity Score (ISS), AIS by body region, and presence of drug or alcohol intoxication]; and clinically relevant times (time to first CTH, time to intervention, and time spent in $\mathrm{ED})$. Intubation in the ED was identified using procedure codes and times. Although the level of TTA is not coded in TQIP, patients were considered to have met standard TTA criteria if they had field $\mathrm{SBP}<90 \mathrm{mmHg}$ and/or field GCS $<9$.

Patients were dichotomized into study groups based on time to first CTH: immediate $\mathrm{CTH}$, defined as $\leq 1 \mathrm{~h}$ after ED arrival, vs. delayed CTH, defined as 1-6 h after ED arrival. The primary outcomes were time to neurosurgical intervention (craniotomy or intracranial pressure [ICP] monitor insertion) and length of time in the ED. Secondary outcomes were in-hospital mortality, hospital length of stay (LOS, days), intensive care unit (ICU) LOS, ventilator days, and need for blood transfusion in the first $24 \mathrm{~h}$.

Descriptive statistics were used to summarize patient characteristics, clinical data, and injury data, with continuous variables presented as median [interquartile range (IQR)] and categorical variables as number (percentage). Univariate analysis compared these parameters between study groups using the Mann-Whitney $U$ test for continuous variables and Chi-square $\left(\chi^{2}\right)$ test for categorical variables.

Multivariate analysis with logistic regression was performed to identify independent factors associated with mortality. Clinically relevant variables and those with $p<0.2$ on univariate analysis were included in the model. Model fit was assessed using the Hosmer-Lemeshow test with results $>0.05$, indicating good fit. Tolerance was $>0.1$ for all independent variables with a variance inflation factor of $<10.0$. Results are expressed as odds ratios (OR) with $95 \%$ confidence intervals (CI). Statistical significance was defined as $p<0.05$. Data were collected and analyzed using IBM SPSS Statistics 23 (IBM Corporation; Armonk, NY).

\section{Results}

Over the study period, 4997 patients with moderately depressed GCS on ED arrival met inclusion and exclusion criteria (Fig. 1). Of these, 3954 (79\%) underwent immediate CTH and 1053 (21\%) underwent delayed CTH. Median time to first CTH was $0.5 \mathrm{~h}$ [IQR 0.3-0.8] in the immediate group and $1.6 \mathrm{~h}$ [IQR $1.3-2.1]$ in the delayed group $(p<0.001)$ (Table 1). More patients in the immediate CTH group satisfied standard TTA criteria $(n=834,21 \%$ vs. $n=133,13 \%$, $p<0.001)$.

Patient demographics, comorbidities, and drug or alcohol intoxication generally did not vary between study groups (Table 1). Mechanism of injury was significantly different between groups $(p<0.001)$, with motor vehicle collisions and motorcycle collisions more common in the immediate CTH group (16\% vs. $9 \%$ and 5\% vs. $3 \%$, respectively). Falls were the most common mechanism of injury in both groups.

GCS was slightly lower in the field among patients who underwent immediate CTH [11 (IQR 9-13) vs. 12 (IQR $10-14), p<0.001$ ], although initial GCS in the ED was 11 [IQR 10-12] in both groups. Head injury severity was not different between groups [Head AIS 4 (IQR 3-4) vs. 4 (IQR $3-4), p=0.586]$. Associated injury severity did not differ between groups in any body regions $(p>0.05)$. ISS was comparable between groups [16 (IQR 10-21) vs. 16 (IQR $10-21), p=0.079]$.

Univariate analysis of outcomes revealed no difference in need for craniotomy ( $11 \%$ vs. $10 \%, p=0.287)$, need for ICP monitor ( $12 \%$ vs. $12 \%, p=0.899)$, or mortality $(11 \%$ vs. $9 \%, p=0.160$ ) between groups (Table 2). Patients who received immediate $\mathrm{CTH}$ had a shorter time from ED arrival to craniotomy [3.1 h (IQR 2.1-8.7) vs. $4.2 \mathrm{~h}$ (IQR 3.0-7.6), $p=0.001$ ] but no difference in time from CTH to 


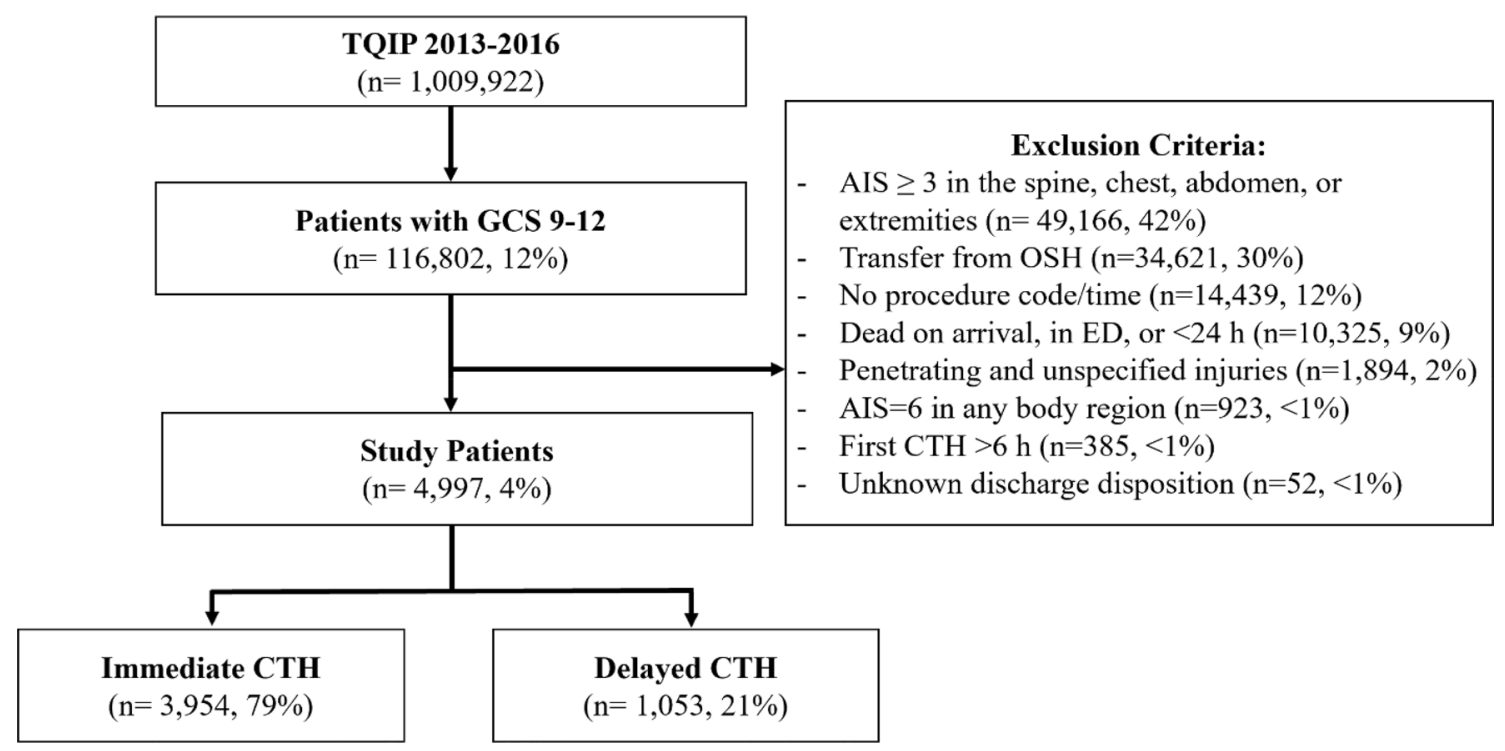

Fig. 1 Flow of Patients through Study. TQIP, Trauma Quality Improvement Program. GCS, Glasgow Coma Scale score. AIS, Abbreviated Injury Scale score. OSH, outside hospital. ED, emer-

craniotomy [2.8 h (IQR 1.7-8.5) vs. $2.6 \mathrm{~h}$ (IQR 1.6-11.6), $p=0.632]$. The same was true for ICP monitor insertion, with patients who received immediate $\mathrm{CTH}$ having a shorter time to ICP monitor placement $[4.4 \mathrm{~h}$ (IQR 2.6-12.0) vs. $5.7 \mathrm{~h}(3.8-13.0), p=0.008$ ] but similar time from first $\mathrm{CTH}$ to ICP monitor placement [3.9 h (IQR 2.0-11.0) vs. $3.6 \mathrm{~h}$ (IQR 1.8-11.0), $p=0.806$ ]. Patients undergoing immediate $\mathrm{CTH}$ were in the ED for a shorter period of time $[2.1 \mathrm{~h}$ (IQR $1.2-3.7)$ vs. $4.3 \mathrm{~h}$ (IQR $2.7-6.5), p<0.001$ ).

Multivariate analysis of risk factors for in-hospital mortality revealed that age $>65$ (OR 2.813, $p<0.001$ ), $\mathrm{SBP}<90 \mathrm{mmHg}$ on arrival (OR 2.934, $p=0.004)$, ED intubation (OR 1.486, $p=0.001$ ), and Head AIS scores of 4 (OR $1.884, p<0.001)$ and 5 (OR 6.729, $p<0.001$ ) were independently associated with increased risk of death (Table 3 ). Higher GCS score upon ED arrival (OR 0.900, $p=0.025$ ) and alcohol (OR 0.504, $p<0.001$ ) or drug intoxication (OR $0.557, p<0.001)$ were associated with reduced mortality.

\section{Discussion}

The appropriate triage of trauma patients is a critical process both for optimal patient outcome and hospital resource utilization. The ACS COT has defined a list of TTA criteria that are applied in the field and identify trauma patients at the highest risk for mortality $[1,3]$. TTA triggers early mobilization of personnel and resources, designed to facilitate expedited diagnosis and treatment of these severely injured patients. The desire to provide immediate maximal care is balanced, however, with hospital resource utilization. The gency department. CTH, computed tomography scan of the head. Immediate $\mathrm{CTH}, \leq 1 \mathrm{~h}$ after ED arrival. Delayed CTH, 1-6 h after ED arrival.

ideal TTA criteria will allow prehospital identification of all at-risk patients without resource activation for low-risk patients. The rate of undertriage, or identification of highrisk, severely injured patients not identified by TTA criteria, is carefully monitored. This is used as a variable in developing center-specific modifications, such as the addition of geriatric age [4-6], to the criteria set forth by the ACS COT.

Excessive levels of overtriage are undesirable because of the potential strain on resource utilization. Undertriage, however, presents a patient safety concern, and for that reason, acceptable rates are significantly lower. Previous studies have demonstrated that patients who are undertriaged are at increased risk of mortality compared to patients who are not undertriaged [7, 8]. The specific mechanisms by which undertriage contributes to or is associated with increased mortality are unknown. Theoretically, delayed diagnosis or intervention may be expected when personnel and resources are not mobilized prior to patient arrival.

One of the resources that is commonly mobilized with the highest level TTA is expedited access to CT scan. Because the management of TBI hinges upon evaluation with crosssectional imaging, time to CT scan among patients with TBI may play a role in outcomes. After undertriage, without prioritized access to cross-sectional imaging, patients with depressed GCS appear to be at particular risk for adverse outcomes and need for intervention. One study demonstrated that the elevated mortality risk after undertriage was the highest among patients with initial ED GCS $<9$ [8]. Another study demonstrated that undertriaged patients with ED GCS $<11$ are at high risk for in-hospital mortality and need for emergent surgical intervention [2]. This study 
Table 1 Patient demographics, clinical data, and injury data

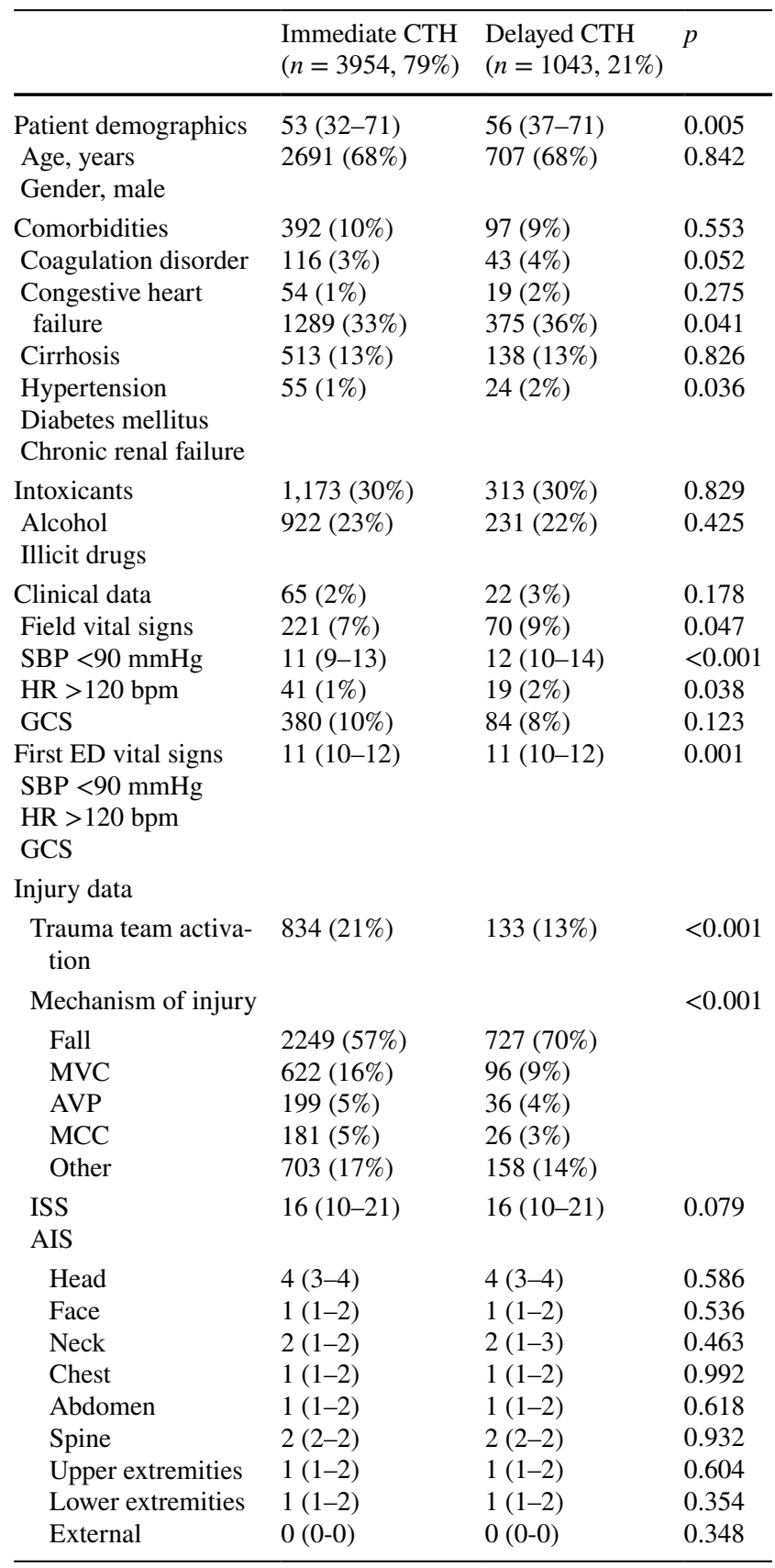

Continuous variables presented as median [interquartile range]. Categorical variables presented as number (percentage) Trauma team activation, defined by patients with field $\mathrm{SBP}<90 \mathrm{mmHg}$ and/or field GCS $<9$

$C T H$ computed tomography scan of the head, SBP systolic blood pressure (mmHg), $H R$ heart rate (beats per minute), GCS Glasgow coma scale score, $M V C$ motor vehicle collision, $A V P$ auto vs. pedestrian collision, $M C C$ motorcycle collision, ISS injury severity score, $A I S$ abbreviated injury scale score

also suggested that shifting the TTA trigger for GCS score to $<11$ from the current $<9$ would have a minimal impact on the population overtriage rate as these patients tend to be severely injured.

Because patients with moderately depressed GCS do not trigger prehospital TTA under the existing ACS COT criteria, the significance of this neurologic perturbation may initially be minimized. The potential etiologies of moderately altered levels of consciousness are also broad. Early underestimation of injury burden in combination with diagnostic confusion can result in a less streamlined process of investigation and intervention for traumatic injuries, which may in turn have an effect on outcomes. This has been demonstrated in other aspects of care of the injured patient. For example, delayed time to hemorrhage control with pelvic angiography for patients with blunt pelvic fractures is associated with increased mortality [9]. Similarly, this study hypothesized that delayed time to CTH may be associated with poor outcomes after TBI.

This study demonstrated that immediate CTH was associated with faster time to disposition out of the ED and shorter time to neurosurgical intervention. The concept of earlier diagnosis expediting determination of ED discharge destination is intuitive and has the potential for far-reaching effects at the hospital level. Studies targeting methods of increasing ED throughput have shown that this reduces ED congestion [10], improves hospital workflow, and is associated with shortened hospital length of stay [11]. Furthermore, increased ED throughput allows for an increase in the volume of patients seen [12], which has implications for hospital earnings. Although the implications for the overall flow of patients through the ED is unknown, resource expenditure to expedite CTH for trauma patients may carry benefits at the hospital level as immediate $\mathrm{CTH}$ allows for faster discharge out of the ED for patients with moderately depressed GCS.

Immediate CTH among patients presenting with moderately depressed GCS was also associated with shorter time to intervention, both with craniotomy and ICP monitor insertion. Among patients who required neurosurgical intervention, the time between $\mathrm{CTH}$ and intervention was the same regardless of whether the patient underwent immediate or delayed CTH. This suggests that the timing of the index $\mathrm{CTH}$ is a rate-limiting step to intervention.

Time to intervention is a critical determinant of survival in many trauma populations $[9,13,14]$. These data did not demonstrate reduced mortality or need for craniotomy among patients undergoing immediate CTH. Mortality was slightly higher among patients undergoing immediate CTH, although the difference was not statistically significant. This may be a result of more severely injured patients undergoing cross-sectional imaging sooner than less severely injured counterparts.

Death or need for surgical intervention may not be the best outcome measures in trauma patients with depressed 
Table 2 Univariate analysis of outcomes

\begin{tabular}{llll}
\hline & $\begin{array}{l}\text { Immediate CTH } \\
(n=3954,79 \%)\end{array}$ & $\begin{array}{l}\text { Delayed CTH } \\
(n=1043,21 \%)\end{array}$ & $p$ \\
\hline Times & & & \\
ED arrival to: & & & \\
First CTH & $0.5(0.3-0.8)$ & $1.6(1.3-2.1)$ & $<0.001$ \\
Craniotomy & $3.1(2.1-8.7)$ & $4.2(3.0-7.6)$ & 0.001 \\
ICP monitor insertion & $4.4(2.6-12.0)$ & $5.7(3.8-13.0)$ & 0.008 \\
ED exit & $2.1(1.2-3.7)$ & $4.3(2.7-6.5)$ & $<0.001$ \\
First CTH to: & & & 0.632 \\
Craniotomy & $2.8(1.7-8.5)$ & $2.6(1.6-11.6)$ & 0.806 \\
ICP monitor insertion & $3.9(2.0-11.0)$ & $3.6(1.8-11.0)$ & 0.160 \\
Mortality & $423(11 \%)$ & $96(9 \%)$ & $<0.001$ \\
ED intubation & $1240(31 \%)$ & $187(18 \%)$ & 0.287 \\
Need for craniotomy & $420(11 \%)$ & $99(10 \%)$ & 0.899 \\
Need for ICP monitor & $475(12 \%)$ & $124(12 \%)$ & 0.868 \\
Need for blood transfusion $<24 h$ & $133(3 \%)$ & $34(3 \%)$ & 0.029 \\
Hospital LOS, days & $13(8-23)$ & $15(9-25)$ & 0.288 \\
ICU LOS, days & $8(5-15)$ & $9(5-14)$ & 0.204 \\
Ventilator days & $5(3-11)$ & $6(2-12)$ & \\
\hline
\end{tabular}

Continuous variables presented as median [interquartile range]. Categorical variables presented as number (percentage). All times are given in hours

$C T H$ computed tomography scan of the head, ED emergency department, ICP intracranial pressure, $L O S$ length of stay, $I C U$ intensive care unit
GCS. The advantages of prompt craniotomy are challenging to quantify as outcomes after TBI are more granular than mortality alone. Functional and cognitive neurologic outcomes may be more clinically meaningful in this patient population. Examination of the impact of rapid surgical intervention on functional and cognitive neurologic recovery after TBI will be the next phase in this study. Overall, existing data would support the concept of improved outcomes with decreased time to intervention.

Taken together, these study findings suggest that time to $\mathrm{CTH}$ is a rate-limiting step in the care of patients who arrive to the ED with a moderately depressed GCS. Resource allocation to allow more rapid access to CTH among these patients could be expected to shorten time to intervention and ED length of stay. This would be beneficial for both patients and busy emergency departments. One method to achieve expedited access to CTH for these patients may be a limited TTA triggered in the ED by a GCS score of 9-12. The limited TTA could consist of prioritized access to the CT scanner without other changes in resource allocation or personnel distribution. Because these patients tend to be severely injured, this would not be expected to change the population overtriage rate in any meaningful way.
This study has several limitations. It is a retrospective, registry-based study and is associated with the inherent restrictions of this type of study design. In particular, there is a lack of granularity that comes from working with registry data. Outcomes, such as functional and cognitive neurologic capacity at discharge, are of interest but are not captured by TQIP. Additionally, specific CTH findings are not delineated in TQIP. Lastly, a type II error must be considered with this study's inability to demonstrate a difference in mortality based on time to CTH. These limitations can be addressed in the future with a large multicenter study using non-registry-based data.

To conclude, patients who arrive to the ED with moderately depressed GCS after trauma are not captured by current ACS COT TTA criteria but are at risk for adverse outcomes. Patients with TBI are likely to benefit from early intervention and those who do not undergo immediate CTH spent more time in the ED and had delayed time to intervention (craniotomy or ICP monitor insertion). Additional resource allocation to expedite $\mathrm{CTH}$ for blunt trauma patients who have a depressed GCS but do not meet TTA criteria may therefore be beneficial for both patients and hospitals. 
Table 3 Multivariate analysis of independent risk factors for mortality

\begin{tabular}{llll}
\hline & \multicolumn{2}{l}{ Mortality } & \\
\cline { 2 - 4 } & Adj $p$ value & OR & $95 \%$ CI \\
\hline Age $>65$ & $<0.001$ & 2.813 & $(2.275-3.479)$ \\
Gender, male & 0.532 & 0.936 & $(0.760-1.152)$ \\
Intoxicants & & & \\
Alcohol & $<0.001$ & 0.504 & $(0.375-0.677)$ \\
Illicit drugs & $<0.001$ & 0.557 & $(0.408-0.762)$ \\
ED vital signs & & & \\
SBP $<90$ mmHg & 0.004 & 2.934 & $(1.412-6.097)$ \\
HR $>120$ bpm & 0.565 & 1.105 & $(0.786-1.555)$ \\
GCS & 0.025 & 0.900 & $(0.820-0.987)$ \\
Trauma team activation & 0.765 & 1.040 & $(0.805-1.344)$ \\
Head AIS & & & \\
$=1,2,3$ & REF & 1.884 & $(1.375-2.581)$ \\
=4 & $<0.001$ & 6.729 & $(4.928-9.187)$ \\
5 & $<0.001$ & & \\
Immediate CTH & 0.519 & 1.087 & $(0.844-1.399)$ \\
Need for craniotomy & 0.393 & 0.881 & $(0.659-1.178)$ \\
ED intubation & 0.001 & 1.486 & $(1.188-1.860)$ \\
\hline
\end{tabular}

Multivariate analysis with logistic regression. Test for collinearity was performed prior to analysis. AUROC 0.792 (95\% CI $0.773-$ 0.812). Trauma team activation, defined by field $\mathrm{SBP}<90 \mathrm{mmHg}$ and/or field GCS $<9$. Immediate CTH, computed tomography scan of the head $\leq 1 \mathrm{~h}$ of ED arrival

$A d j$ adjusted, $O R$ odds ratio, $C I$ confidence interval, $E D$ emergency department, SBP systolic blood pressure $(\mathrm{mmHg}), H R$ heart rate (beats per minute), GCS Glasgow coma scale score, AIS abbreviated injury scale score

Funding No funding was received for this study.

\section{Compliance with ethical standards}

Conflict of interest All the authors declare that they have no conflict of interest.

Ethical approval All procedures performed in this retrospective observational study involving human participants were in accordance with the ethical standards of the Institutional Review Board of the University of California (HS-19-00632) and with the 1964 Helsinki declaration and its later amendments or comparable ethical standards.

Informed consent This study received a waiver for informed consent by the Institutional Review Board of the University of Southern California (HS-19-00632).

\section{References}

1. American college of surgeons committee on Trauma (ACS COT). (2014) Resources for optimal care of the injured patient, 6th Ed.
Chicago, Il: American college of surgeons. https://www.facs.org/-/ media/files/quality-programs/trauma/vrc-resources/resources-foroptimal-care.ashx.

2. Schellenberg M, Benjamin E, Bardes JM, Inaba K, Demetriades D. Undertriaged trauma patients: who are we missing? J Trauma. 2019;87(4):865-9.

3. Davis JW, Dirks RC, Sue LP, Kaups KL. Attempting to validate the overtriage/undertriage matrix at a level I trauma center. J Trauma. 2017;83(6):1173-8.

4. Bardes JM, Benjamin E, Schellenberg M, Inaba K, Demetriades D. Old age with a traumatic mechanism of injury should be a trauma team activation criterion. J Emerg Med. 2019;57(2):151-5.

5. Cull J, Riggs R, Riggs S, Byham M, Witherspoon M, Baugh N, Metcalf A, Kitchens D, Manning B. Development of trauma level prediction models using emergency medical service vital signs to reduce over- and undertriage rates in penetrating wounds and falls of the elderly. Am Surg. 2019;85(5):524-9.

6. Benjamin ER, Khor D, Cho J, Biswas S, Inaba K, Demetriades D. The age of undertriage: current trauma triage criteria underestimate the role of age and comorbidities in early mortality. J Emerg Med. 2018;55(2):278-87.

7. Polites SF, Leonard JM, Glasgow AE, Zielinski MD, Jenkins DH, Habermann ER. Undertriage after severe injury among United States trauma centers and the impact on mortality. Am J Surg. 2018;216(4):813-8.

8. Tignanelli CJ, Vander Kolk WE, Mikhail JN, Delano MJ, Hemmila MR. Noncompliance with American college of surgeons committee on trauma recommended criteria for full trauma team activation is associated with undertriage deaths. J Trauma. 2018;84(2):287-94.

9. Matsushima K, Piccinini A, Schellenberg M, Cheng V, Heindel P, Strumwasser A, Benjamin E, Inaba K, Demetriades D. Effect of door-to-angioembolization time on mortality in pelvic fracture: every hour of delay counts. J Trauma. 2018;84(5):685-92.

10. Beck MJ, Okerblom D, Kumar A, Bandyopadhyay S, Scalzi LV. Lean intervention improves patient discharge times, improves emergency department throughput, and reduces congestion. Hosp Pract. 1995;44(5):252-9.

11. Hag N, Stewart-Corral R, Hamrock E, Perin J, Khalig W. Emergency department throughput: an intervention. Intern Emerg Med. 2018;13(6):923-31.

12. Garrett JS, Berry C, Wong H, Qin H, Kline JA. The effect of vertical split-flow patient management on emergency department throughput and efficiency. Am J Emerg Med. 2018;36(9):1581-4.

13. Kotwal RS, Howard JT, Orman JA, Tarpey BW, Bailey JA, Champion HR, Mabry RL, Holcomb JB, Gross KR. The effect of a golden hour policy on the morbidity and mortality of combat casualties. JAMA Surg. 2016;151(1):15-24.

14. Meizoso JP, Ray JJ, Karcutskie CA 4th, Allen CJ, Zakrison TL, Pust GD, Koru-Sengul T, Ginzburg E, Pizano LR, Schulman CI, et al. Effect of time to operation on mortality for hypotensive patients with gunshot wounds to the torso: the golden 10 minutes. J Trauma. 2016;81(4):685-91. 